\title{
Producción de alimentos para animales a través de fermentación en estado sólido - FES
}

\author{
Animal feed production by solid state fermentation - SSF \\ Produção De Alimentos Para Animais A Través De Fermentação Em \\ Estado Sólido - FES
}

\section{Luis M. Borrás-Sandoval ${ }^{1}$, Giovanny Torres-Vidales ${ }^{2}$}

1 Zootecnista, MSc, PhD (c), Universidad pedagógica y Tecnológica de Colombia, Facultad Ciencias Agropecuarias, Grupo de Investigación GIBNA, Tunja, Colombia.

2 MVZ, Esp, Universidad pedagógica y Tecnológica de Colombia, Facultad Ciencias Agropecuarias, Grupo de Investigación GIDIMEVEZ, Tunja, Colombia.

Email: Lumibo30@yahoo.es

Recibido: septiembre 02 de $2013 \quad$ Aceptado: agosto 30 de 2016

\section{Resumen}

La Fermentación en Estado Sólido (FES), se consolida como una alternativa para la alimentación animal, gracias a este proceso biotecnológico los residuos de cosecha y desechos agroindustriales se pueden convertir en alimentos energético -proteicos, de alto valor nutricional que en un momento dado sustituyan total o parcialmente los alimentos balanceados, que encarecen sensiblemente los costos de producción, haciendo cada vez menos rentables las explotaciones pecuarias, es por esto que la FES, se convierte no solo en una alternativa económicamente viable, sino ambientalmente sostenible, a partir del manejo de residuos de alto potencial contaminante. La presente revisión, busca mostrar la FES como una alternativa biotecnológica para la obtención de alimentos que pueden ser utilizados en la producción animal.

Palabras clave: Fermentación en estado sólido, Biotecnología, Alimento animal.

\begin{abstract}
Solid state fermentation (SSF) has become an alternative for animal feed; this biotechnological process means that harvesting residues and agro-industrial waste can be converted into energy-providing-protein-based feed having high nutritional value which, at any given moment, will totally or partially substitute balanced foods which significantly increase production costs, making livestock farms less profitable. SSF thus becomes not just an economically viable alternative but also an environmentally sustainable one by managing residues having highly contaminating potential. This review has been aimed at highlighting SSF as a biotechnological alternative for obtaining feed which can be used in animal production.
\end{abstract}

Key words: solid state fermentation, biotechnology, animal food.

\section{Resumo}

A fermentação em estado sólido (FES), está se tornando numa ótima opção para o uso de resíduos vegetais e resíduos agro-industriais, graças a este processo biotecnológico podem ser convertidos em alimentos energéticos e proteícos de 
alto valor nutricional, num determinado momento pode substituir total ou parcialmente os alimentos balanceados, que aumentam os custos de produção fazendo tornar significativamente explorações pecuárias menos rentáveis, por isso a FES, torna-se não só numa alternativa viável economicamente, mas a gestão de resíduos ambientalmente sustentável com elevado potencial poluente. A presente revisão tem como objetivo mostrar uma alternativa para a obtenção de alimentos biotecnológicos a serem utilizados na produção animal

Palavras-chave: Fermentação em estado sólido, Biotecnologia, Alimentação animal.

\section{Introducción}

La alimentación animal, históricamente ha competido por materias primas con la alimentación humana, ya que algunas de ellas como el maíz, el sorgo, la soya, etc. Se constituyen en la base de los alimentos concentrados y es allí donde se genera la rivalidad entre estos; en este momento, a dicha disputa entra un tercer actor y son los biocombustibles, los cuales se basan para su producción en muchos de estos cereales y granos, escaseándolos y por ende encareciéndolos; por tal razón, es necesario plantear alternativas de alimentación animal basada en fuentes y alimentos no convencionales (residuos de cosecha, materiales fibrosos, desechos de la industria, etc.) y es aquí, donde opciones biotecnológicas como las fermentaciones y en especial la fermentación en estado sólido (FES), se consolidan como una alternativa para la generación de alimentos proteico-energéticos de buena calidad y de bajo costo, esto necesariamente mejorará la rentabilidad del agronegocio ganadero y en general de las producciones pecuarias, dando una alternativa económicamente viable y ambientalmente sostenible, si se piensa en estos residuos como posibles contaminantes ambientales si no se les da un manejo adecuado.

La fermentación es un proceso catabólico de oxidación incompleta, con alto grado de anaerobiosis, siendo el producto final un compuesto orgánico. Estos productos finales son los que caracterizan los diversos tipos de fermentaciones.

La fermentación fue descubierta por Louis Pasteur, que la describió como la vie sans I'air (la vida sin el aire). Es un proceso catabólico de oxidación incompleta, totalmente anaeróbico, siendo el producto final un compuesto orgánico. Estos productos finales son los que caracterizan los diversos tipos de fermentaciones. La fermentación típica es llevada a cabo por las levaduras, sin embargo algunos metazoos y protistas son capaces de realizarla. (Mena, 2013).

\section{Tipos de fermentación dependiendo su producto final.}

Fermentación acética, es la fermentación bacteriana por Acetobacter, un género de bacterias aeróbicas que trasforman el alcohol en ácido acético. La formación de ácido acético $(\mathrm{CH} 3 \mathrm{COOH})$ resulta de la oxidación de un alcohol por la bacteria del vinagre en presencia de oxígeno. Estas bacterias a diferencia de las levaduras productoras de alcohol requieren de un suministro generoso de oxígeno para su funcionamiento. (Resendiz, 2009).

Fermentación alcohólica, transformación de azúcares en etanol y $\mathrm{CO}_{2}$, por levaduras, como Saccharomyces cerevisiae, durante la elaboración de vinos, cervezas y similares (Zanabria, 2009).

Fermentación láctica, la fermentación láctica da como resultado ácido láctico a partir de la degradación de la lactosa. De igual manera se produce en las células musculares cuando existe una deficiencia de oxígeno en los músculos; a partir de ácido pirúvico. (Carmona et al., 2010).

Fermentación Butírica, es la conversión de los glúcidos en ácido butírico, por acción de bacterias de la especie Clostridium Butirycus. Se produce a partir de la lactosa, con formación de ácido butírico y gases como dióxido de carbono, metano ácidos grasos volátiles e hidrogeno. Se caracteriza por la aparición de olores pútridos y desagradables. (Ocampo, 2011).

Estas biotécnicas funcionan con bacterias, hongos filamentosos, levaduras y algas unicelulares. Sus avances se soportan en la manipulación de estos microorganismos, que son útiles para la producción de alimentos, medicamentos y otros productos. Específicamente las bacterias desarrollan una gran capacidad de producción de proteínas; las fermentaciones microbianas tienen la ventaja de utilizar poca energía, dado que la fermentación puede utilizar procedimientos que no requieren altas temperaturas. (Ramos et al., 2007).

La biotecnología es una herramienta importante para el desarrollo sostenible de la agricultura, la pesca y la actividad forestal, así como de las industrias alimentarias. Cuando se integra debidamente con otras tecnologías para la producción de alimentos, productos agrícolas y servicios, puede contribuir en gran medida a satisfacer, en el nuevo milenio, las necesidades de una población en crecimiento y cada vez más urbanizada. 
El Convenio sobre la diversidad biológica (CDB) define la biotecnología como "toda aplicación tecnológica que utilice sistemas biológicos y organismos vivos o sus derivados para la creación o modificación de productos o procesos para usos específicos". (FAO, 2000).

\section{Fermentación en Estado Sólido}

La fermentación en estado sólido (FES) consiste en hacer crecer un microorganismo sobre un sustrato, empleando una fuente de nitrógeno y sales mineralizadas (ricas en macro y micronutrientes), bajo ciertas condiciones de humedad, $\mathrm{pH}$, aireación y temperatura. La FES no presenta agua libre en su estructura, aunque conlleva determinados requerimientos de humedad (Doelle et al., 1992; Echavarría et al., 2003).

Onteru et al., (2010), resaltan los procesos biotecnológicos desarrollados en los países en desarrollo lo que han llamado la "revolución ganadera", dentro de ellos la FES para la producción de enzimas fibrolíticas, utilizadas en la producción de alimentos para animales. Como antecedentes la FES, es un proceso antiguo que se ha utilizado especialmente en la obtención de productos como panes, quesos, kojí, shoyu y bebidas como el Sake. Además Pastrana (1996), cita a (Kumar y Losane, 1989) quienes reportan aplicaciones industriales de los cultivos en estado sólido entre las que se encuentran la producción de enzimas, ácidos orgánicos, toxinas, antibióticos y otros metabolitos de interés. Ajila et al., (2012), presentan los residuos agrícolas e industriales, como una fuente importante de biomasa para ser utilizada en la alimentación animal, por procesos como la FES, la cual enriquece estos residuos y los hace más digestibles para los animales.

Pandey et al., (2000), Singhaniaa et al., (2009), manifiestan que estos procesos fermentativos se consideraban décadas atrás como de baja tecnología, hoy en día son una alternativa muy prometedora, no solo para la producción de alimento animal sino con muchos otros usos, farmacéuticos, bioremediación, biodegradación de compuestos peligrosos, etc.

\section{Ventajas e inconvenientes de los procesos de fermentación en estado sólido}

Pastrana (1996), enumera una serie de ventajas de los procesos generales de la fermentación en estado sólido (FES) entre las que se incluyen:

- Los medios de cultivo son simples, generalmente subproductos agrícolas que presentan un alto contenido de los nutrientes necesarios para el proceso fermentativo.
- Fermentadores con menores requerimientos espaciales, ya que los sustratos se utilizan más concentrados y no se utilizan grandes volúmenes de agua.

- La baja actividad del agua es de gran ayuda para evitar las contaminaciones, especialmente de bacterias y levaduras.

- Mayor simplicidad en el diseño de los fermentadores y en los sistemas de control.

- La aireación forzada es facilitada por la porosidad del soporte, lo que permite alta transferencia de oxígeno al microorganismo.

- Mayores facilidades para la obtención y aplicación del inóculo, pudiendo utilizarse las esporas directamente en la mayor parte de las situaciones.

- Facilidad para el escalado de los procesos.

- Necesidades reducidas de disolventes para la extracción de los productos.

- Rendimientos comparables, e incluso superiores, a los correspondientes procesos en cultivo sumergido.

- El proceso de recobrado es simplificado, ya que algunos productos son utilizados integralmente como alimento animal,

- Reducido riesgo de contaminación bacteriana, menos aptas para soportar la baja actividad de agua que caracteriza a estos sistemas. Posibilidad en ocasiones, de trabajar incluso en condiciones no asépticas.

- Elevada aireación del sistema, lo que hace a esta modalidad de cultivo especialmente adecuada a aquellos procesos que impliquen un metabolismo oxidativo intenso.

- Bajos requerimientos energéticos. A menudo no es preciso esterilizar, airear ni agitar.

- Ambiente similar al de los hábitats naturales de los microorganismos utilizados.

- Reducido volumen de efluentes.

- Los procesos se consideran generalmente como tecnologías limpias.

Entre las desventajas inherentes al sistema debe mencionarse lo siguiente:

- Frecuente necesidad de pretratamiento de los sustratos (molienda y prehidrólisis parciales). 
- Su aplicación se limita a microorganismos que crecen en bajos contenidos de humedad.

- Dificultad para mantener los niveles óptimos de humedad durante la fermentación.

- La extracción del calor metabólico puede ser un problema, sobre todo cuando se trabaja a gran escala y no se controla el proceso.

- Ausencia de métodos analíticos simples para determinar el crecimiento microbiano.

- Dificultad para la agitación en aquellos procesos que así lo requieran.

- Frecuente necesidad de inóculo voluminoso.

- Los procesos de transferencia de masa son limitados por la difusión.

- Aspectos ingenieriles como el diseño de reactores y el escalado están muy poco caracterizados.

\section{Alimentos producidos por medio del proceso de fermentación en estado sólido (FES), para alimentación animal.}

La utilización de la FES se consolida en la producción de alimento para animales, utilizando diversas materias primas para este fin, si bien es cierto, se destaca que el proceso fermentativo ha dado origen a diversos alimentos desde tiempos remotos, la utilización de este tipo de tecnologías en la alimentación animal, es más reciente y hoy frente a los diversos escenarios de escases y encarecimiento de materias primas tradicionales utilizadas en la alimentación humana y animal, se convierte en gran alternativa para este fin, en especial, si se tiene en cuenta la posibilidad de uso de otras fuentes no convencionales para la alimentación animal, como los residuos de cosecha, materiales con alto contenido fibroso y bajo nivel nutricional, todos ellos gracias a las bondades de la fermentación en estado sólido, se pueden transformar en alimentos económicos y de gran valor nutricional. A continuación se describen algunos desarrollos realizados de amplio uso en la alimentación de diversas especies animales con evidentes resultados zootécnicos y de producción animal.

\section{La Saccharina como punto de partida para la elaboración de alimentos para uso animal elaborados por FES}

Elías et al., (1990), reportan la elaboración de un alimento bajo la tecnología de fermentación en estado sólido, el cual, tuvo como objeto el enriquecimiento proteico de la caña de azúcar y que gracias a la FES, lograron un producto proteico-energético, de gran importancia para la alimentación animal, el cual se denominó Saccharina. Se reporta que este producto presenta valores en proteína bruta similares al maíz y el trigo y valores de energía bruta de $16 \mathrm{MJ} / \mathrm{kg} \mathrm{MS}$. Los niveles de fibra varían del 25 al 30\%, destacándose que la mayor parte está formada por celulosa y hemicelulosa. Es importante destacar que la caña utilizada es limpia sin hojas ni cogollo. También se han realizado trabajos con caña integral (tallos, hojas y cogollo) con resultados similares, es decir, con calidad nutricional muy parecida a la Saccharina elaborada con la caña limpia (Vivas y Carvajal, 2004; Monroy et al., 2006). Torres et al., (2007) realizaron una variación en la elaboración de Saccharina utilizando tallos quemados de caña, sin encontrar diferencias en la calidad nutricional.

Zarragoitia et al., (1990), reportaron la utilización de la Saccharina y la Leucaena (Leucaena leucocephala) como suplemento a hembras bovinas (Holstein) en crecimiento, en pastizales de gramíneas de secano, en este trabajo se obtuvo ganancias diarias de peso promedio de $735 \mathrm{gr} /$ día, con un concentrado que contenía 58\% de Saccharina en la dieta.

En cuanto a la utilización de la Saccharina en otros animales de importancia zootécnica, se presenta el trabajo, "Consumo y digestibilidad en carneros alimentados con diferentes proporciones de Saccharina en el concentrado", elaborado por Ruiz et al., (1990). En este trabajo se sustituye maíz y trigo en dietas de carneros por Saccharina, en porcentajes de inclusión de esta hasta el $90 \%$ en la dieta, incrementando la digestibilidad de la fibra bruta, hasta $62 \%$, además, se reporta que la energía suministrada con adición del $70 \%$ de Saccharina en la dieta, solo representó una diferencia de tan solo $5 \%$ con respecto a concentrado de granos (maíz, trigo). Lo anterior, valida la posibilidad de utilizar altas proporciones de Saccharina en dietas de rumiantes. Al respecto cabe anotar lo encontrado por Aguirre et al., (2010), quienes al alimentar ovinos con distintos tipos de caña de azúcar entre ellas las fermentadas, destacan que efectivamente los residuos de caña fermentados mejoraron significativamente su valor nutricional, pero no mejoran la respuesta zootécnica de los animales.

Diversos autores han publicado trabajos en varias especies animales, donde se puede evidenciar diferentes niveles de sustitución de alimentos concentrados por Saccharina sin afectación en los parámetros productivos (Tabla 1). 
Tabla 1. Niveles de Sustitución de alimento concentrado por Saccharina en diferentes especies.

\begin{tabular}{|l|c|l|}
\hline \multicolumn{1}{|c|}{ Especie } & \multicolumn{1}{c|}{$\begin{array}{c}\text { (\%) de inclusión de } \\
\text { Saccharina en la dieta }\end{array}$} & \multicolumn{1}{c|}{ Autores } \\
\hline Gansos (reproductores) & 20 & Valdivié et al., 1990 \\
\hline Gansos mantenimiento y 30 y 60 días & 60 & Valdivié et al., 1990 \\
\hline Gansos primeros 30 días & 30 & Valdivié et al., 1990 \\
\hline Pavos después de los 45 días al sacrificio & 30 & González et al., 1993 \\
\hline Patos primeros 30 días & 10 & Fraga et al., 1994 \\
\hline Patos 30 días al sacrificio & 20 & Fraga et al., 1994 \\
\hline Pollos de ceba & 10 & Valdivié and Elias, 2006 \\
\hline Gallinas pesadas & 10 a 20 & Valdivié et al., 1997 \\
\hline Gallinas livianas & 10 & Valdivié et al., 1993 \\
\hline Gallinas de guinea & 20 & Fraga et al., 1993 \\
\hline Cerdos precebos & 10 & Lezcano et al., 1990 \\
\hline Cerdos ceba & 20 & Castro et al., 1990 \\
\hline Cerdas gestantes & 40 & Díaz et al.,, 1991 \\
\hline Cerdas lactantes & 20 & Díaz et al., 1991 \\
\hline Conejos & 10 & Valdivié et al., 2012 \\
\hline
\end{tabular}

Adaptada. Valdivié et al., 2012

\section{Preparados microbianos} para el enriquecimiento de FES

Se ha encontrado que al inocular preparados microbianos en los procesos de FES se evidencia una mejora en la digestibilidad y en la calidad nutricional de los alimentos producidos bajo esta tecnología. Hsu, et al.,
(2013), utilizaron la FES, para tratar forrajes (elefante y pangola), inoculando bacterias celulolíticas se encontró que estas enriquecían el contenido proteico de las gramíneas y mejoraron la digestibilidad en los pollos. En la tabla 2, se puede observar diferentes trabajos con la utilización de preparados microbianos para el mejoramiento de FES.

Tabla 2. Elaboración de alimentos por medio de fermentación en estado sólido Inoculados con preparados microbianos (Bacterias, Hongos, levaduras).

\begin{tabular}{|c|c|c|}
\hline Producto & Resultados & Autores \\
\hline $\begin{array}{l}\text { Bagazo caña + Tricoderma viride } \\
\text { M5-2 }\end{array}$ & $\begin{array}{l}20 \% \text { más de digestibilidad del bagazo } \\
\text { y } 11 \% \text { de proteína cruda. }\end{array}$ & Valiño et al., 2004 \\
\hline Caña de azúcar + Pleurotus sapidus & $\begin{array}{l}\text { Mejora digestibilidad y fermentación ruminal } \\
\text { en novillas en condiciones tropicales }\end{array}$ & Peláez et al., 2008 \\
\hline $\begin{array}{l}\text { Cotiledón de soya - granos de destilería } \\
\text { +Aspergullus oryzae, Trichoderma } \\
\text { reesei, Phanerochaete chrysosporium }\end{array}$ & $\begin{array}{l}\text { Disminución en la fibra bruta y } \\
\text { aumento en la proteína cruda }\end{array}$ & Lio y Wang, 2012 \\
\hline Tamos de trigo + Ganoderma $s p$ & $\begin{array}{l}\text { Aumento en la digestibilidad y calidad } \\
\text { nutricional para rumiantes }\end{array}$ & Shrivastava, et al., 2011 \\
\hline Caña de azúcar + Rodotorula & $\begin{array}{l}\text { Encontró a las } 48 \text { de inoculación } \\
\text { mayor enriquecimiento proteico }\end{array}$ & Rodríguez, et al., 2006 \\
\hline Platano + Lentinus crinitus & $\begin{array}{l}\text { El producto fermentado no solo tiene potencial de } \\
\text { uso en la alimentación animal sino farmacéutico }\end{array}$ & Granda et al., 2005 \\
\hline Caña de azúcar + Pleurotus sapidus & $\begin{array}{l}\text { Mejor en la digestibilidad de la materia seca } \\
\text { y las variables fermentativas por medio de } \\
\text { la generación de enzimas fibrolíticas }\end{array}$ & Peláez, et al., 2011 \\
\hline Cáscara de cacao + Pleurotus $s p$ & $\begin{array}{l}\text { Disminuye hasta en un } 50 \% \text { la materia orgánica } \\
\text { e incrementa la PB por encima del } 13 \%\end{array}$ & Bermúdez et al., 2002 \\
\hline
\end{tabular}

Fuente: los autores. 


\section{Otros alimentos obtenidos por FES}

Otras modificaciones en la obtención de alimentos por medio de FES, es incluir diferentes materias primas y por medio del proceso obtener alimentos que puedan ser utilizados en la alimentación animal.

El desecho de la Uva, también ha sido utilizado en FES, Berradre et al., (2009), utilizaron el desecho de la industria vinícola, empleando el bagazo de la Uva, con unos resultados muy interesantes ya que el producto final de la fermentación tiene un potencial grandísimo, en la elaboración de abonos orgánicos y no por ello, se descarta la utilización en la alimentación animal, por encima de esto vale la pena resaltar el beneficio ambiental generado por FES, en el manejo de residuos los cuales potencialmente, se pueden convertir en un problema ambiental muy grande.

El plátano, también se ha trabajado como sustrato en la fermentación en estado sólido, Granda et al., (2005), muestran las posibilidades de trabajar el plátano utilizando el hongo Lentinus crinitus, para obtener productos con potencial de uso no solo en la alimentación animal, sino con aplicaciones de uso farmacéutico, esto nos muestra el amplio espectro de utilización del proceso biotecnológico de la fermentación en estado sólido, FES.

Rodríguez et al., (2010), proponen una alternativa de FES en residuos de manzana, enriquecida con diferentes niveles de soya y urea, con muy buenos resultados de incremento en la proteína del alimento, al tener un excelente crecimiento bacteriano y de levaduras en los residuos de manzana.

Ramos, et al., (2006) utilizaron la caña de azúcar enriquecida con residuales de sorgo (saccha-sorgo) y con pulidura de arroz (saccha-piludo) obteniendo un alimento de mejor composición bromatológica con disminución de las fracciones de fibra. Cárdenas et al., (2008), desarrollaron un alimento por FES utilizando Sorgo y bagacillo de caña obteniendo una disminución en el contenido de fibra y un incremento hasta en $11 \%$ de la proteína verdadera en el producto final.

Trabajos recientes han utilizado residuales de papa con harina de alfalfa para obtener un alimento por FES, el cual disminuyo la cantidad de fibra del alimento mejorando la digestibilidad, logrando un alimento con 21\% de proteína de la cual el $70 \%$ es proteína verdadera (Borrás et al., 2014). Wang et al., (2010), exponen como la pulpa de papa tratada con FES puede ser utilizada en la alimentación de las aves sin mostrar efectos negativos.

Finalmente, otros trabajos plantean la utilización de materias primas no convencionales para la elabo- ración de alimentos por FES, como es el caso de la utilización del nopal el cual fue fermentado y secado para posteriormente ser incorporado en bloques multinutricionales en dietas de ovinos, observándose gran aceptabilidad por estos sin mostrar diferencias significativas sobre los parámetros productivos (Mejía et al., 2011). Igualmente se obtuvo un alimento a base de la harina del fruto del árbol del pan (Artocarpus altilis) con diferentes inclusiones de urea, obteniendo incremento en la síntesis de proteína microbiana y disminuyendo la materia seca del alimento (Brea et al., 2015).

\section{Conclusiones}

Los países tropicales como Colombia, con gran diversidad de climas, que tienen vastos territorios dedicados a la producción de gran variedad de frutas, hortalizas y cultivos de cereales, gramíneas y leguminosas que generan residuales especialmente de cosecha y de la agroindustria. Esta gran biomasa de residuos tiene un potencial uso en la alimentación animal, pero al no ser utilizada se puede convertir en un factor contaminante del medio ambiente. Por lo tanto con procesos biotecnológicos sencillos como la fermentación en estado sólido (FES), se puede lograr transformar dichos residuos en alimentos con altos niveles energético-protéicos, económicos, de gran valor nutricional y ambiental para que sean suministrados a diversas especies animales.

\section{Bibliografía}

Aguirre J, Magaña R, Martínez S, Gómez A, Ramírez J, Barajas R, Plascencia A, Barcena R y García D. Caracterización nutricional y uso de la caña de azúcar y residuos transformados en dietas para ovinos, Zootecnia Trop. 2010; 28(4):489-497.

Ajila C, Brar S, Verma M, Tyagi RD, Godbout S y Valéro J. Bio-processing of agro-byproducts to animal feed, Crit Rev Biotechnol. 2012; 32(4):382-400.

Berradre M, Mejías M, Ferrer J, Chandler C, Páez G, Mármol Z, Ramones $\mathrm{E}$, y Fernández V. Fermentación en estado sólido del desecho generado en la industria vinícola, Rev Fac Agron. (LUZ). 2009; 26:398-422.

Borras L, Elias A, Moyano M. Efecto de la Temperatura y el Tiempo sobre los Indicadores de la Papa (Solanum tuberosum) Fermentada en Estado Sólido. Ciencia y Agricultura. 2014;11(2):31-38.

Brea O, Elías A, Ortiz A, Motta W, Hechavarría S. Efecto de la urea y del tiempo en la fermentación en estado sólido de la harina del fruto del árbol del pan ( Artocarpus altilis). Ciencia y Agricultura. 2015; 12(2):84-91.

Cárdenas J, Aranda E, Hernández D, Lagunes L, Ramos J y Salgado S. Obtención de un alimento fermentado en estado sólido a partir del bagacillo de retorno, pulido de arroz e inóculos. Su 
utilización en la alimentación animal, Rev Cubana Cienc. Agríc. 2008; 42(2):173-176.

Carmona Irving et al., 2010. Fermentación láctica. Disponible en: http://www.slideshare.net/PREPA2600C/fermentacion-lacticax-d2, consultado marzo de 2013.

Castro M, Martínez M. La alimentación porcina con productos no tradicionales: cincuenta años de investigación en el instituto de ciencia animal. Rev Cubana Cienc Agríc. 2015;49(2):180-196

Castro M, Díaz J, Lezcano P, Elías A, Iglesias M. Sistemas de alimentación para cerdos en ceba con dietas basadas en miel B y pienso con Saccharina. Rev Cubana Cienc Agríc. 1990;24:91-93.

Díaz J, Torres A, Rodríguez Y. Miel final y piensos con Saccharina para cerdas en desarrollo. Rev Cubana Cienc Agríc. 1991; 25:289-292.

Echavarría J, López P, Mato S. Alternativas para la alimentación animal utilizando fermentación en estado sólido, Revista Avanzada Científica. 2003;6(1):1-13.

Elías A, Lezcano O, Lezcano P, Cordero J, Quintana L. Reseña descriptiva sobre el desarrollo de una tecnología de enriquecimiento proteico en la caña de azúcar mediante fermentación en estado sólido (Saccharina), Rev. Cubana Cien. Agric. 1990, 24:1-6.

FAO declaración sobre biotecnología. 2000. Disponible en: http:// www.fao.org/biotech/fao-statement-on-biotechnology/es/, consultado marzo de 2013.

Fraga L M, Valdivié M, Elias A and Rodríguez C. Feeding of broilers with Saccharina o Leucasaccharina. Cuban J. Agric. Sci. $1993 ; 27: 63$

Fraga LM, Valdivié M, Febles M, Gutiérrez M, Rodríguez C. A note on the utilization of Saccharina in the fattening of White Peking ducks. Cuban J. Agric. Sci. 1994;28:347.

González LM, Valdivié M, Lon-Wo E, Elías A, Rodríguez J, Gutiérrez $\mathrm{O}$. A note on the use of industrial Saccharina in turkey fattening. Cuban J. Agric. Sci. 1993; 27:69

Granda R, Diana M, Mejía G, Amanda I, Jiménez T, Gloria A. Utilización de residuos de plátano para la producción de metabolitos secundarios por fermentación en estado sólido con el hongo, Lentinus crinitus, vitae, revista de la facultad de química farmacéutica. 2005;12(2):13-20.

Hsu PK, Liu CP, LiuL Y, Chang CH, Yang SS. Protein enrichment and digestion improvement of napiergrass and pangolagrass with solid-state fermentation, J Microbiol Immunol Infect. 2013;46 (3):171-179.

Lezcano P, Elias A, Lamazares E, Achan J. Niveles de inclusión de Saccharina en el pienso para cerdos en preceba, Rev Cubana Cien Agric. 1990;24:85-88.

Lio J, Wang T. Solid-state fermentation of soybean and corn processing coproducts for potential feed improvement, J Agric Food Chem. 2012;60(31): 7702-7709.

Mejía J, Delgado J, Mejía I, Guajardo I, Valencia M. Efectos de la suplementación con bloques multinutricionales a base de nopal fermentado sobre la ganancia de peso de ovinos en crecimiento, Acta Universitaria. 2011; 21(1):11-16.
Mena C. 2013. Procesos fermentativos, disponible en: http:// es.scribd.com/doc/15762395/Procesos-Fermentativos, consultado marzo de 2013.

Monroy J, Aranda E, Mendoza G, Ramos J, Herrera J, Cobos M, Izquierdo F. Elaboración y conservación de Saccharina a partir de caña de azúcar integral, con la adición de melaza y pulidura de arroz. Rev. Cubana Cien. Agric. 2006;40(2): 167-172.

Ocampo de Jesús. et al., 2011. Microfermentación. Disponible en: http://www.slideshare.net/iwannalike/microfermentacion, consultado marzo de 2013.

Onteru S, Ampaire A y Rothschild M. Biotechnology developments in the livestock sector in developing countries. Biotechnol Genet Eng Rev. 2010;27:217-228.

Pandey A, Soccol C, Mitchell D. New developments in solid state fermentation: I-bioprocesses and products, Process Biochemistry. 2000;35:1153-1169.

Pastrana L. Fundamentos de la fermentación en estado sólido y aplicación a la industria alimentaria. Ciencia y Tecnología Alimentaria. 1996;1(3):4-12.

Peláez A, Meneses M, Miranda L, Megías M, Barcena R, Loera O. Ventajas de la fermentación sólida con pleurotus sapidus en ensilajes de caña de azúcar, Archivos de Zootecnia. 2008;57(217):25-33.

Peláez A, Meneses M, Miranda L, Ayala M Crosby M, Loera O, Megias-Rivas D. Enzimas fibrolíticas producidas por fermentación en estado sólido para mejorar los ensilajes de caña de azúcar. Agrociencia. 2011;45:675-685.

Ramos J, Elías A Herrera F. Procesos para la producción de un alimento energético - proteico para animales. Efecto de cuatro fuentes energéticas en la fermentación en estado sólido (FES) de la caña de azúcar, Rev. Cubana Cien. Agric. 2006;40(1);51-58.

Ramos J, Elías A, Herrera F, Aranda E, Mendoza G. Procesos para la producción de un alimento energético-proteico para animales. Efecto de niveles de miel final en la fermentación en estado sólido del Saccha-sorgo y Saccha-pulido, Rev. Cubana Cien. Agric. 2007;41(2):139-143.

Resendiz Yamili et al., 2009. Fermentación, disponible en: http:// www.slideshare.net/lucas1111/fermentacion-22, consultado Marzo de 2013.

Rodríguez C, Díaz D, Salvador F, Ruiz O, Arzola C, Flores A, Elias A. Efecto del nivel de urea y pasta de soya en la Concentración de proteínas durante la fermentación en estado sólido de manzana de desecho (Malus domestica), Rev. Cubana Cien. Agric. 2010;44(1):24-26.

Rodríguez ZR, Boucourt R, Elías A, Madera M. Dinámica de fermentación de mezclas de caña (Saccharum officinarum) y boniato (Ipomea batata), Rev. Cubana Cien. Agric. 2001;35(2):147-151.

Rodríguez Z, Boucourt R, Elías A, Herrera F, Nuñez O. Efecto del grosor de la capa en la dinámicade fermentación de mezclas de caña (Saccharum officinarum) y boniato (Ipomea batata Lam), Rev. Cubana Cien. Agric. 2006; 40(2):173-182.

Ruiz R, Cairo J, Marrero D, Elias A. Consumo y digestibilidad en carneros alimentados con diferentes proporciones de 
Saccharina en el concentrado, Rev. Cubana Cien. Agric. 1990;24:61-64.

Shrivastava B, Nandal P, Sharma A, Jain KK, Khasa YP, Das TK, Mani V, Kewalramani NJ, Kundu SS, Kuhad RC. Solid state bioconversion of wheat straw into digestible and nutritive ruminant feed by Ganoderma sp. rckk02, Bioresour Technol. 2011;107:34751.

Singhaniaa R, Patelb A, Soccolc C, Pandeya A. Recent advances in solid-state fermentation, Biocheml Eng J. 2009;44(1):3-18.

Torres N, Aranda E, Mendoza G, Hernández D, Hernández AL. Landois $L$, Ramos J. Consumo y producción de leche de vacas de doble propósito, suplementadas con Saccharina elaborada con caña de azúcar quemada, Rev. Cubana Cien. Agric. 2007;41(3):223-226.

Valdivié BM, Bernal BH. (2012). Alimentación de aves, cerdos y conejos. Con yuca, batata, banano, arroz, caña, DDGS y Amaranto. Ed. Universidad autónoma de león, Capitulo IX, pág. 149-168.

Valdivie M, Elias A, Dieppa O. Alimentación de gansos con Saccharina. 1. Etapa de ceba. Rev. Cubana Cien. Agric. 1990a; 24: 97-102.

Valdivie M, Elias A, Dieppa O. Alimentación de gansos con Saccharina. 2. Adaptación a altas concentraciones. Rev. Cubana Cien. Agric. 1990b;24:103-107.

Valdivie M, Elias A, Dieppa O. Alimentación de gansos con Saccharina. 3. Ceba con Saccharina elaborada a partir del bagacillo de retorno. Rev. Cubana Cien. Agric. 1990c;24:207-212.
Valdivie M, Elias A, Dieppa O. Utilización de la Saccharina en los piensos para pollos de engorde. Rev. Cubana Cien. Agric. 1990d;24:109-112.

Valdivié M, González M, Elías A. Nuevos tipos de Saccharina para aves. Revista Cubana de Ciencia Agrícola. 1997;31(3):231-236.

Valdivié M. and Elías A. Potentialities of the Canavalia ensiformis grain fermented with sugarcane (Sacchacanavalia) in broilers. Cuban J. Agric. Sci. 2006;40:441.

Valiño E, Elías A, Torres V, Carrasco T, Albelo N. Mejoramiento de la composición del bagazo de caña de azúcar por la cepa Trichoderma viride M5-2 en un biorreactor de fermentación en estado sólido. Rev. Cubana Cien. Agric. 2004;38(2):145153.

Vivas N, Carvajal J. Saccharina rustica una aplicación biotecnológica para la alimentación animal, revista Facultad de Ciencias Agropecuarias. $2004 ; 2(1): 43-48$.

Wang T, Wu Y, Jiang C, Liu Y. Solid state fermented potato pulp can be used as poultry feed. Br Poult Sci. 2010; 51(2):229-34.

Zanabria Aldo. 2009. Fermentaciones industriales, disponible en: http://www.slideshare.net/aldozpuno/f-e-r-m-e-n-t-a-c-i-o-n-e-s-i, consultado marzo de 2013.

Zarragoitia I, Elias A, Ruiz T, Plaza J, Rodriguez J. Utilización de la Saccharina y la Leucaena (Leucaena leucocephala) como suplemento a hembras bovinas en crecimiento en pastizales de gramíneas de secano, Rev. Cubana Cien. Agric. 1990; 24-43. 